\title{
An Interactive Music Playlist Generator that Responds to User Emotion and Context
}

\author{
Darryl Griffiths \\ Glyndŵr University \\ Mold Road \\ Wrexham LL11 2AW \\ UK \\ griffiths.d@glyndwr.ac.uk
}

\author{
Stuart Cunningham \\ Glyndŵr University \\ Mold Road \\ Wrexham LL11 2AW \\ UK \\ s.cunningham@glyndwr.ac.uk
}

\author{
Jonathan Weinel \\ Glyndŵr University \\ Mold Road \\ Wrexham LL11 2AW \\ UK \\ j.weinel@glyndwr.ac.uk
}

\section{INTRODUCTION}

This paper aims to demonstrate the mechanisms of a music recommendation system, and accompanying graphical user interface (GUI), that is capable of generating a playlist of songs based upon an individual's emotion or context. This interactive music playlist generator has been designed as part of a broader system, Intended for mobile devices, which aims to suggest music based upon 'how the user is feeling' and 'what the user is doing' by evaluating real-time physiological and contextual sensory data using machine learning technologies. For instance, heart rate and skin temperature in conjunction with ambient light, temperature and global positioning satellite (GPS) could be used to a degree to infer one's current situation and corresponding mood.

At present, this interactive music playlist generator has the ability to conceptually demonstrate how a playlist can be formed in accordance with such physiological and contextual parameters. In particular, the affective aspect of the interface is visually represented as a two-dimensional arousalvalence space based upon Russell's circumplex model of affect (1980).Context refers to environmental, locomotion and activity concepts, and are visually represented in the interface as sliders. These affective and contextual components are discussed in more detail next in Sections 2 and 3 , respectively. Section 4 will demonstrate how an affective and contextual music playlist can be formed by interacting with the GUI parameters. For a comprehensive discussion in terms of the development of this research, refer to (Griffiths et al. 2013a, 2013b, 2015). Moreover, refer to Teng et al. (2013) and Yang et al. (2008) for related work in these broader research areas.

\section{AFFECTIVE CONCEPTS}

The underlying mechanism that informs the emotion part of the interface is based upon Russell's circumplex model of affect, which is a generally accepted model of emotion. This model theoretically indicates that emotions differ in their level of similarity and that some are usually perceived as contrasting emotions (see Figure 1).

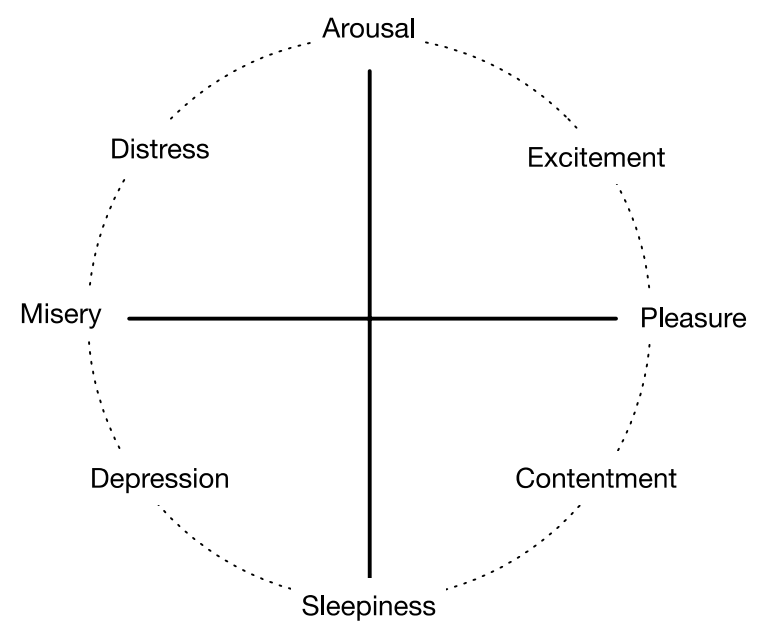

Figure 1: Russell's circumplex model of affect

\section{CONTEXTUAL CONCEPTS}

The contextual part of the system is based upon environment, motion and activity concepts (see Table 1).

Table 1: Contextual concepts

\begin{tabular}{|c|l|l|l|l|}
\hline \multicolumn{2}{|c|}{ Environment } & \multicolumn{2}{c|}{ Locomotion } & \multicolumn{1}{c|}{ Activity } \\
\hline Dark & Light & $\begin{array}{l}\text { Slow } \\
\text { Static }\end{array}$ & $\begin{array}{l}\text { Fast } \\
\text { Active }\end{array}$ & $\begin{array}{l}\text { Run Indoors } \\
\text { Cycle Indoors } \\
\text { Relax Indoors }\end{array}$ \\
\hline
\end{tabular}




\section{GENERATING MUSIC PLAYLISTS}

This shows how affective and contextual playlists are created by interacting with the system.

\subsection{Generating an affective music playlist}

Affective music playlists can be generated by simply selecting a point in space to express how one feels. It is anticipated that emotion detection will be inferred automatically using real-time physiological sensory data from the user. Figure 2 demonstrates the manner in which an affective music playlist can be generated by selecting a single point around the middle area of the fourth quadrant. Music in the system's database is preanalysed based upon previous research findings that describes the relationship between audio features and emotional response, which allows each track to be located on the circumplex model. The red circle visually indicates a cluster of musical tracks that are in close proximity to the selected point using a Euclidean distance metric.

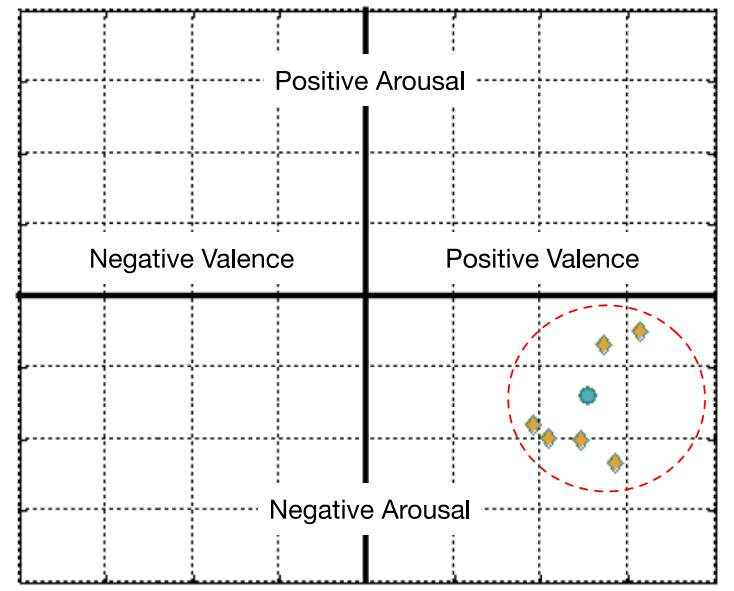

Figure 2: A computational model of affect

Table 2 shows selected songs from this point, and their respective ordering.

Table 2: Affective song selection and ordering

\begin{tabular}{|l|l|l|}
\hline No. & \multicolumn{1}{|c|}{ Song } & \multicolumn{1}{c|}{ Genre } \\
\hline 1 & Take Five & Jazz \\
\hline 2 & Oh It Is Jesus & Gospel \\
\hline 3 & Chan Chan & World \\
\hline 4 & Oxygene & Electronic \\
\hline
\end{tabular}

\subsection{Generating a contextual music playlist}

Contextual music playlists can be generated by adjusting sliders that correspond to the environmental, locomotion and activity concepts. The former two sliders are bi-polar, which indicates that the middle point is a neutral state. In contrast, the activity sliders are uni-polar. All of the sliders can be adjusted to the extent to which these concepts are present to select music playlists.
However, they can only be operated in isolation at this moment. It is intended that these parameters will controlled through the use of automatic context detection in future work. For example, the degree of light reported from an ambient light sensor may be inferred as 'dark' (see Figure 3).

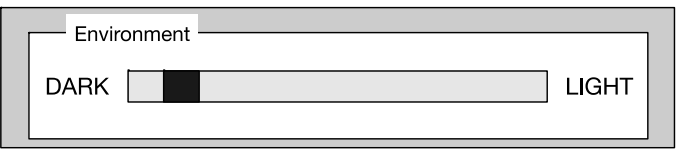

Figure 3: The environment slider reporting 'dark'

Table 3 shows some of the selected songs from this slider position, and their respective ordering.

Table 3: Song selection and ordering for 'dark'

\begin{tabular}{|l|l|l|}
\hline No. & \multicolumn{1}{|c|}{ Song } & \multicolumn{1}{c|}{ Genre } \\
\hline 1 & Adagio For Strings & Classical \\
\hline 2 & Passage De Recherche & Avant Garde \\
\hline 3 & For Whom The Bell Tolls & Heavy Metal \\
\hline 4 & I Wanna Be Adored & Independent \\
\hline
\end{tabular}

The activity-based concepts could also be interpreted by evaluating multiple channels of sensory data, such as ambient light, temperature, location from GPS, accelerometer movement, etc.

Work is on-going to determine how best to allow these processes to interact, since both emotion and context concepts operate in isolation. Validation is underway of the affective recommendation system.

\section{REFERENCES}

Griffiths, D., Cunningham, S., and Weinel, J. (2013a) Automatic Music Playlist Generation Using Affective Computing Technologies. ITA13, September 10-13, pp. 177-183.

Griffiths, D., Cunningham, \& S., Weinel, J. (2013b) A Discussion of Musical Features For Automatic Music Playlist Generation Using Affective Technologies. AM13, September 16-20.

Griffiths, D., Cunningham, S., and Weinel, J. (2015) A Self-Report Study Which Gauges Perceived and Induced Emotion With Music. ITA15, September 711, pp. 236-241.

Russell, J. A. (1980) A Circumplex Model of Affect. Journal of Personality and Social Psychology, vol. 39, no. 6, pp. 1161-1178.

Teng, Y. C., Kuo, Y. S., Yang, Y. H., and Chen, H. (2013) A Large In-Situ Dataset For Context-Aware Music Recommendation For Smartphones. ICMEW, July 15-19, pp. 1-4.

Yang, Y. H., Lin, Y. C., Cheng, H. T., and Chen, H. (2008) Mr. Emo: Music Retrieval in the Emotion Plane. MM ‘08, October 26, pp. 1003-1004. 